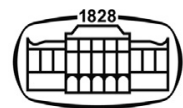

AKADÉMIAI KIADÓ

\title{
A pilot study on the pharmacokinetics of a single intramuscular injection of cefquinome in Arabian camel calves
}

\section{Acta Veterinaria Hungarica}

68 (2020) 1, 59-64

DOI:

10.1556/004.2020.00008

๔ 2020 Akadémiai Kiadó, Budapest

\section{ORIGINAL ARTICLE}

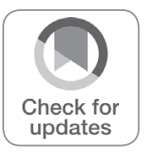

\author{
ABDULLAH ALTAYBAN ${ }^{1}$, MAHMOUD KANDEEL $^{1,2 *}$ (1), \\ YUKIO KITADE ${ }^{3,4}$ and MOHAMMED AL-NAZAWI ${ }^{1}$
}

\author{
${ }^{1}$ Department of Physiology, Biochemistry and Pharmacology, King Faisal University, Al Hofuf, Al \\ Ahsa, 31982, Saudi Arabia \\ ${ }^{2}$ Department of Pharmacology, Faculty of Veterinary Medicine, Kafrelshiekh University, \\ Kafrelshiekh, Egypt \\ ${ }^{3}$ Department of Applied Chemistry, Faculty of Engineering, Aichi Institute of Technology, \\ Yachigusa, Yakuza, Toyota, Japan \\ ${ }^{4}$ Department of Chemistry and Biomolecular Science, Faculty of Engineering, Gifu University, \\ Yanagido, Gifu, Japan
}

Received: March 29, 2019 • Accepted: September 25, 2019

Published online: May 8, 2020

\section{ABSTRACT}

This study was conducted to evaluate the pharmacokinetics of cefquinome in camel calves after a single intramuscular injection in a dose of $2 \mathrm{mg} / \mathrm{kg}$ body weight ( $\mathrm{kg} \mathrm{b}$. w.). Cefquinome concentrations were measured by ultra-high performance liquid chromatography-mass spectrometry (UPLC-MS/MS). A non-compartmental pharmacokinetic model was used to fit the time-concentration curve and estimate the pharmacokinetic parameters. The peak serum concentration $\left(\mathrm{C}_{\max }\right)$ was $28.4 \mu \mathrm{g} / \mathrm{mL}$ at the time of maximum concentration $\left(\mathrm{T}_{\max }\right)$ of $25 \mathrm{~min}$. The elimination half-life $\left(\mathrm{t}_{1 / 2}\right)$ was $17.4 \mathrm{~h}$. The area under the concentration-time curve $\left(\mathrm{AUC}_{0-\infty}\right)$ was $103.7 \mu \mathrm{g} / \mathrm{ml}^{-1} \mathrm{~h}$ and the mean residence time $\left(\mathrm{MRT}_{0-\infty}\right)$ was $21.3 \mathrm{~h}$. In comparison with other animal species, the pharmacokinetics of cefquinome in Arabian camel calves showed faster absorption from the site of injection and slower elimination. Since cefquinome, as other beta-lactams, is a time-dependent antimicrobial agent, a single dose of $2 \mathrm{mg} / \mathrm{kg} \mathrm{b}$. w. might be sufficient against the most sensitive organisms in camel calves owing to its prolonged elimination phase. However, dose readjustment is required for cases needing concentrations above $2 \mu \mathrm{g} / \mathrm{mL}$ for $12 \mathrm{~h}$ or above $1 \mu \mathrm{g} / \mathrm{mL}$ for $24 \mathrm{~h}$.

\section{KEYWORDS}

cefquinome, camel, pharmacokinetics, UPLC-MS/MS, cephalosporins

\section{INTRODUCTION}

Cephalosporins are an important class of therapeutic agents with wide applications in veterinary medicine (Caprile, 1988; Hornish and Katarski, 2002; Wang et al., 2017). Cefquinome is a fourth-generation cephalosporin with proven broad-spectrum activity against Grampositive and Gram-negative bacteria including Escherichia coli (Zhao et al., 2017), Klebsiella pneumonia (Qu et al., 2018), Actinobacillus pleuropneumoniae (Zhang et al., 2018), Pasteurella (Zhang et al., 2019), and Staphylococcus aureus (Yu et al., 2016).

Owing to the wide range of microorganisms susceptible to cefquinome, this drug is highly recommended for treating complex infections during mastitis (Yu et al., 2016; Bachmann et al., 2018), endometritis (Amiridis et al., 2003), respiratory infections (Funaki et al., 2001), and septicaemia (Thomas et al., 2004). The pharmacokinetics of cefquinome were previously estimated in sheep (Corum et al., 2019), rabbits (Elazab et al., 2018), buffalo calves

*Corresponding author. Tel.: 00966 568918734.

E-mail: mkandeel@kfu.edu.sa; mahmoud.kandeal@vet.kfs.edu.eg 
(Venkatachalam et al., 2018b), slider turtles (Uney et al., 2018), swans (Zhao et al., 2017), horses (Uney et al., 2017), beagle dogs (Zhou et al., 2015), and fish (Shan et al., 2015). Although the pharmacokinetics of cefquinome has been studied in several animal species, its applications and pharmacokinetic parameters in camel calves have not been determined. The aim of this study was to determine the pharmacokinetics of cefquinome in order to enable its proper therapeutic use in Arabian camel calves.

\section{MATERIALS AND METHODS}

\section{Animals and facilities}

Three male Arabian camel calves (age: 4 months, body weight: $100 \mathrm{~kg}$ ) were used in this study. The camels were kept in the premises of the Camel Research Centre, King Faisal University. Twelve hours before the experiment, food was withdrawn. Drinking water was freely available.

\section{Chemicals and drugs}

All animal procedures were approved by the Ethics Committee of King Faisal University (approval no. KFU-REC/ 2018-11-2). Cefquinome (Cobactan 2.5\%, MSD Animal Health, Cairo, Egypt) was injected intramuscularly at a dose rate of $2 \mathrm{mg} / \mathrm{kg}$ b. w.; a similar dose had been used in buffalo calves (Venkatachalam et al., 2018a) as well as sheep and goats (El-Hewaity et al., 2014). Acetonitrile, methanol and ammonium acetate for UPLC were purchased from SigmaAldrich (St. Louis, MO, USA). The product was approved for the treatment of pneumonia in cattle and pigs as well as mastitis-metritis-agalactia syndrome in pigs.

\section{Collection of samples}

Venous blood samples were collected from the left jugular vein in $10-\mathrm{mL}$ tubes immediately before injection of the drug and $0,5,10,15,30 \mathrm{~min}$ and 1, 2, 4, 6, 12 and $24 \mathrm{~h}$ after drug administration. Blood samples were immediately centrifuged and the serum was stored at $-80{ }^{\circ} \mathrm{C}$ until the determination of cefquinome concentration.

\section{Instrumentation and chromatographic conditions}

Ultra-high performance liquid chromatography (UPLC-MS/ MS) was performed using a Waters Acquity UPLC system (Waters Corp., MA, USA) consisting of a Waters Acquity BEH C18 column, an autosampler and a quaternary solvent management system. Mass spectrometry was performed using Waters Acquity Micromass triple-quadrupole MS quadrupole supplied with an electrospray ion source (Micromass, Manchester, UK), under the control of a MassLynx 4.1 software. The multiple reaction monitoring (MRM) mode of the mass spectrometer was implemented with a positive electrospray ionisation (ESI+) source. The quantitative ion was from the most abundant ion. Cefquinome was confirmed from the other ions (Table 1). The setting of nebulisation and cone gas was $800 \mathrm{~L} / \mathrm{h}$ at $450{ }^{\circ} \mathrm{C}$ and $50 \mathrm{~L} / \mathrm{h}$ with source and dissolution temperatures of $150{ }^{\circ} \mathrm{C}$ and $450{ }^{\circ} \mathrm{C}$, respectively. The capillary voltage was $3.2 \mathrm{kV}$. Detection was carried out in MRM mode for each compound as adapted from Hou et al. (2013), UCT Application Note (2014) and Wang et al. (2017). The collision gas was argon with a collision cell pressure of 0.11 PSI.

Sample preparation was performed as described previously (Legrand et al., 2016; Wang et al., 2017) with slight modifications. Stock standard solutions $(25 \mu \mathrm{g} / \mathrm{mL}$ in water $)$ of cefquinome were prepared and stored at $-30{ }^{\circ} \mathrm{C}$. A standard mix solution containing cefquinome at $5 \mu \mathrm{g} / \mathrm{mL}$ was then prepared by dilution in MilliQ water. Calibration standards were prepared for cefquinome in the following final concentrations: $3.125,6.25,12.5,25,37.5$ and 150 $\mathrm{ng} / \mathrm{mL}$.

The sample was separated on a Waters Acquity ${ }^{\mathrm{TM}} \mathrm{BEH}$ C18 column $(2.1 \times 50 \mathrm{~mm}, 1.7 \mu \mathrm{m})$. The gradient elution using mobile phase A was composed of $0.1 \%$ formic acid in water containing $0.5 \mathrm{mM}$ ammonium acetate, while mobile phase $\mathrm{B}$ consisted of $0.1 \%$ formic acid in methanol containing $0.5 \mathrm{mM}$ ammonium acetate. A flow rate of 0.30 $\mathrm{mL} / \mathrm{min}$ was maintained for the analysis time of $4 \mathrm{~min}$. The gradient elution program was set to start with $85: 15$ solutions $\mathrm{A}: \mathrm{B}(\mathrm{v} / \mathrm{v})$ and deliver 15:85 A:B (v/v) within $4 \mathrm{~min}$.

The method was validated for selectivity, sensitivity, linearity, precision, accuracy and stability. The selectivity of the method was evaluated by comparing the blank samples of the chromatograms and the samples with cefquinome. Sensitivity applies for LLOQ (lower limit of quantification), where the response of LLOQ was more than five times higher than the blank at the same retention time of cefquinome. Detection was carried out in MRM mode adapted from Hou et al. (2013), UCT Application Note 5109-02-01 (2014) and Legrand et al. (2016). Using the signal-to-noise method, the peak-to-peak noise around cefquinome retention time was measured, then the value of noise to signal ratios was estimated for each compound by the autointegrator of the instrument software. A signal-to-noise ratio $(\mathrm{S} / \mathrm{N})$ of three is generally accepted for estimating LOD and a $\mathrm{S} / \mathrm{N}$ of ten is used for estimating LOQ. For linearity, several concentrations of cefquinome were prepared to contain

Table 1. Multiple reaction monitoring (MRM) transition parameters of cefquinome

\begin{tabular}{lcccccc}
\hline Compound & Precursor & Q1/CE & Q2/CE & Cone voltage & Q2/Q1 ratio & RT \\
\hline Cefquinome & 529 & $134 / 25$ & $125 / 35$ & 20 & 16.47 & 2.03 \\
\hline
\end{tabular}

Q1: quantitative ion; Q2: qualitative ion; CE: collision energy; RT: retention time. 
$0.195 \mathrm{ppb}-150 \mathrm{ppb}$. These solutions were analysed and the peak areas were calculated. The calibration curve was plotted using peak area vs. concentration of the standard solutions. The obtained correlation coefficient was $r=0.998686, \mathrm{r}^{2}=$ 0.997373 , and the calibration curve was $499.822 * \mathrm{x}+$ -117.284 . The precision applies to by intra- and inter-day precision, which includes six replicates at each LLOQ, low quality control (LQC), middle quality control (MQC) and high quality control (HQC) levels, each on the same analytical run. Inter-assay precision and accuracy were calculated after repeated analysis in three different analytical runs. Accuracy of the method was determined by recovery experiments. The relative recovery of cefquinome compares the recovered amount of cefquinome after adding a known quantity.

Fifty- $\mu \mathrm{L}$ aliquot serum samples were deproteinised with $350 \mu \mathrm{L}$ acetonitrile. After thorough vortex mixing for $1 \mathrm{~min}$, the mixtures were centrifuged at 13,000 rpm for 10 min. Aliquots of the supernatant $(350 \mu \mathrm{L})$ were transferred to glass tubes and evaporated at $40{ }^{\circ} \mathrm{C}$ under a gentle stream of nitrogen. The residues were dissolved in $100 \mu \mathrm{L}$ of the mixture of mobile phase A and B $(9: 1, v / v)$. Finally, $20 \mu \mathrm{L}$ of the solution was injected into the UPLC-MS/MS system.

\section{Pharmacokinetic analysis}

The pharmacokinetic model for the intramuscular injection of cefquinome was tested by nonlinear curve fitting analysis. Several curve-fitting models were implemented, including non-compartment, one- or two-compartment extravascular models. The data were best fitted by a non-compartment model with the aid of PKsolver Excel add-on software (Zhang et al., 2010). The following pharmacokinetic

Table 2. Pharmacokinetic parameters obtained for cefquinome (2 $\mathrm{mg} / \mathrm{kg}$ b. w.) after intramuscular injection to camel calves $(\mathrm{n}=3)$

\begin{tabular}{lcc}
\hline Parameter & Unit & Value (average $\pm \mathrm{SD})$ \\
\hline Lambda_z & $1 / \mathrm{h}$ & $0.040 \pm 0.006$ \\
$\mathrm{t}_{1 / 2}$ & $\mathrm{~h}$ & $17.404 \pm 5.7$ \\
$\mathrm{~T}_{\max }$ & $\mathrm{h}$ & $0.417 \pm 0.1$ \\
$\mathrm{C}_{\max }$ & $\mu \mathrm{g} / \mathrm{mL}$ & $28.387 \pm 8.66$ \\
$\mathrm{C}_{\text {last_obs/C }}$ & & $0.048 \pm 0.007$ \\
$\mathrm{AUC}_{0-\mathrm{t}}$ & $\mu \mathrm{g} / \mathrm{mL} \times \mathrm{h}$ & $69.639 \pm 15.9$ \\
$\mathrm{AUC}_{0-\text { inf_obs }}$ & $\mu \mathrm{g} / \mathrm{mL} \times \mathrm{h}$ & $103.712 \pm 30.07$ \\
AUC $_{0-\mathrm{t} / 0 \text {-inf_obs }}$ & & $0.671 \pm 0.09$ \\
AUMC $_{0-\text { inf_obs }}$ & $\mu \mathrm{g} / \mathrm{mL} \times \mathrm{h}^{2}$ & $2211.022 \pm 914.48$ \\
$\mathrm{MRT}_{0-\text { inf_obs }}$ & $\mathrm{h}$ & $21.319 \pm 5.46$ \\
Vz/F_obs $_{\text {Cl/F_obs }}$ & $(\mathrm{mg}) /(\mu \mathrm{g} / \mathrm{mL})$ & $24.210 \pm 5.6$ \\
\hline
\end{tabular}

Lambda_z: individual estimate of the terminal elimination constant; $\mathrm{t}_{1 / 2}$ : biological half-life; $\mathrm{C}_{\max }$ : peak serum level; $\mathrm{T}_{\max }$ : peak time; AUC: area under serum concentration versus time curve; AUMC: area under the first moment curve; $\mathrm{Cp} 0$ : theoretical serum level at zero time; MRT: mean residence time; Vz/F_obs: apparent volume of distribution during terminal phase after non-intravenous administration. parameters were computed: $t_{1 / 2}$, biological half-life time; $\mathrm{C}_{\text {max }}$, peak serum level; $\mathrm{T}_{\max }$, peak time; $\mathrm{AUC}$, area under the serum concentration versus time curve; $C_{p 0}$, theoretical serum level at zero time; MRT, mean residence time; and $\mathrm{Cl}$, clearance rate (Table 2). Since MIC values of cefquinome against important pathogens of camel calves are not available, the $\mathrm{MIC}_{50}$ values from other animal species were retrieved (Table 3 ). The obtained serum concentrations from this study at 12 and $24 \mathrm{~h}$ after cefquinome administration were compared with the retrieved MIC $_{50}$ values of bacterial pathogens, to help determine the folds of cefquinome concentrations above the required $\mathrm{MIC}_{50}$.

\section{RESULTS}

The progression of serum dose-concentration curves for cefquinome $(2 \mathrm{mg} / \mathrm{kg} \mathrm{b}$. w. im.) in camel calves is shown in Fig. 1. Following administration, the drug was rapidly absorbed with a $\mathrm{C}_{\max }=28.39 \mu \mathrm{g} / \mathrm{mL}$ and the $\mathrm{T}_{\max }$ was 25 min. The drug concentration gradually declined to about 2 $\mu \mathrm{g} / \mathrm{mL}$ at $12 \mathrm{~h}$. After $12 \mathrm{~h}$ the concentration of cefquinome was almost linear just above $1 \mu \mathrm{g} / \mathrm{mL}$ until the end of the sampling time at $2 \mathrm{~h}$. The pharmacokinetic parameters are shown in Table 2. MRT was $21.319 \mathrm{~h}, \mathrm{AUC}_{0-\mathrm{t}}$ was 69.639, the elimination half-live $\left(t_{1 / 2 \lambda z}\right)$ was 0.7 and the total body clearance was $0.964 \mathrm{~mL} \mathrm{~h}^{-1} \mathrm{mg}^{-1}$. The apparent volume of distribution at steady state during extravascular administration (Vz/F_obs) was 24.2.

\section{DISCUSSION}

The fitting model for cefquinome pharmacokinetics was best expressed by using the two-compartment model in buffalo calves (Venkatachalam et al., 2018a), sheep and goats

Table 3. Calculated relationship of the concentration of cefquinome $\left(2 \mathrm{mg} / \mathrm{kg}\right.$ b. w. IM) with its estimated $\mathrm{MIC}_{50}$ values for selected microorganisms (Murphy et al., 1994)

\begin{tabular}{llcc}
\hline & MIC $_{50}$ & \multicolumn{1}{c}{$\begin{array}{c}12 \mathrm{~h} \\
\text { Cefquinome } \\
\text { concentration/MIC }\end{array}$} \\
\hline $\begin{array}{l}\text { Staphylococcus epidermidis } \\
\quad \text { Staphylococcus aureus }\end{array}$ & 0.5 & 382.90 & 0.27 \\
$\quad$ (oxacillin resistant) & 8 & 23.93 & 4.26 \\
Enterococcus species & 8 & 23.93 & 4.26 \\
Enterococcus faecalis & 4 & 47.86 & 2.13 \\
Acinetobacter species & 1 & 191.45 & 0.53 \\
Pseudomonas aeruginosa & 2 & 95.72 & 1.07 \\
Ampicillin-resistant & 0.5 & 382.90 & 0.27 \\
$\quad$ Haemophilus influenzae & & & \\
Streptococcus pneumoniae & 0.06 & 3190.81 & 0.03 \\
Gram-positive anaerobes & 1 & 191.45 & 0.53 \\
Escherichia coli OXA-1 & 1 & 191.45 & 0.53 \\
Escherichia coli SHV-2 & 16 & 11.97 & 0.53 \\
\hline
\end{tabular}




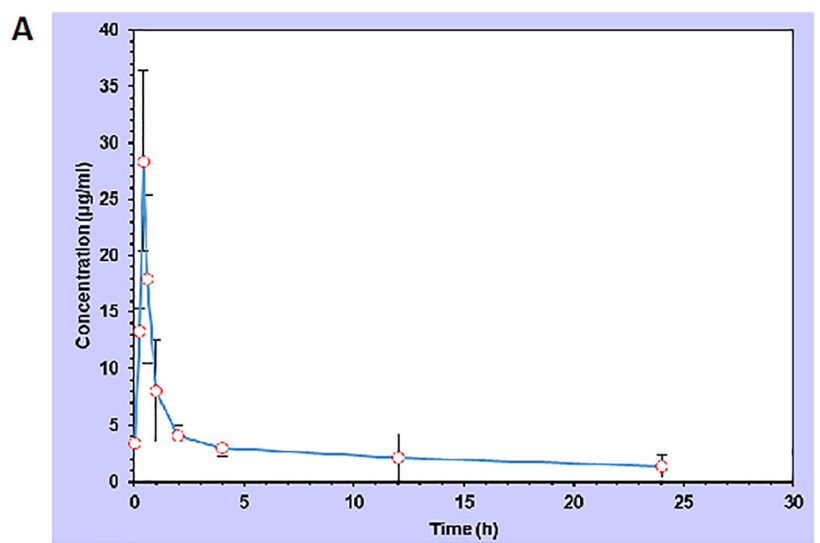

B

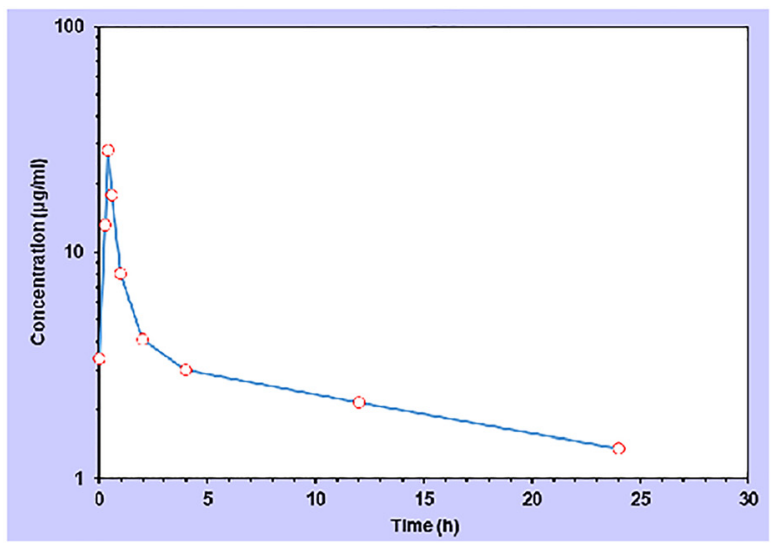

Figure 1. Progression of the dose-response curve (A) and semilogarithmic graph (B) for cefquinome im. injection ( $2 \mathrm{mg} / \mathrm{kg} \mathrm{b}$. w.) in camel calves

(El-Hewaity et al., 2014), the one-compartment model in adult camels (Al-Taher, 2010) and goats (Champawat et al., 2018), and the non-compartmental model in rabbits (Elazab et al., 2017).

The time of maximum serum concentration in this study indicates rapid absorption of cefquinome from the injection site in camel calves as the estimated $\mathrm{T}_{\max }$ was 25 min. This is greatly different from adult camels, which have a $\mathrm{T}_{\max }$ value of $4.25 \mathrm{~h}$ (Al-Taher, 2010). Interestingly, this value is very similar to the $\mathrm{T}_{\max }$ in buffalo calves, which was reported to be $0.5 \mathrm{~h}$ (Venkatachalam et al., 2018a). Adult sheep and goats showed a $\mathrm{T}_{\max }$ of $1.6 \mathrm{~h}$ (El-Hewaity et al., 2014). This might indicate the rapid absorption of cefquinome in camel or buffalo calves compared with adults. Camel calves showed a longer elimination half-life $(17.4 \mathrm{~h})$ than did buffalo calves, sheep and cattle in which this value was $3.56,3.91$ and $1.5 \mathrm{~h}$, respectively (Uney et al., 2011; Dinakaran et al., 2013; Venkatachalam et al., 2018a).

Our study indicates extremely high cefquinome $\mathrm{C}_{\max }$ in camel calves $(28.39 \mu \mathrm{g} / \mathrm{mL})$. This value is higher than that found in sheep ( $4.36 \mu \mathrm{g} / \mathrm{mL}$; Rana et al., 2015), goats (4.83 $\mu \mathrm{g} / \mathrm{mL}$; Dumka et al., 2013) and dogs $(4.83 \mu \mathrm{g} / \mathrm{mL}$; Zhou et al., 2015). This indicates rapid and high absorption of cefquinome from the injection site in camel calves.
The estimated body clearance in camel calves was 0.964 $\mathrm{mL} \mathrm{h}^{-1} \mathrm{mg}^{-1}$, which is higher than reported in other animals such as goats (0.14; Champawat et al., 2018) or healthy rabbits (0.25; Elazab et al., 2018). Similarly, camel calves showed a noticeable increase in MRT $(21.3 \mathrm{~h})$. The MRT was found to be $9.14 \mathrm{~h}$ in sheep (Rana et al., 2015), 14 $\mathrm{h}$ in sheep and $15 \mathrm{~h}$ in goats (El-Hewaity et al., 2014). Interestingly, adult camels showed an MRT of 16.7 (AlTaher, 2010). This suggests that the MRT of cefquinome is generally higher in camels than in other species. The Vz/ F_obs was 24.2. Although $\mathrm{Vz}$ is less competent than $\mathrm{Vd}$ in describing the volume of distribution, it can be used for roughly indicating the general medium distribution of cefquinome in camel calves.

Comparing the pharmacokinetics of cefquinome in camel calves and adult camels, the calves showed a very rapid absorption from the injection site, higher maximal plasma concentration, higher elimination half-life, higher area under curve of drug concentration-time relationship and longer mean residence time.

Cefquinome has a broad spectrum of activity against a wide range of Gram-positive and Gram-negative bacteria (Limbert et al., 1991). Table 3 shows the MIC $_{50}$ of cefquinome against some selected microorganisms (Murphy et al., 1994). The time-concentration curve produced in this study was used to calculate the number of times of serum cefquinome concentrations above the $\mathrm{MIC}_{50}$ after 12 and $24 \mathrm{~h}$. After $12 \mathrm{~h}$, cefquinome had a serum concentration 11-3190 times higher than the estimated $\mathrm{MIC}_{50}$ values. In this context, the level of cefquinome at $12 \mathrm{~h}$ was about 11.97 times higher than the $\mathrm{MIC}_{50}$ for E. coli SHV-2 and 3190.81 times higher than the $\mathrm{MIC}_{50}$ for Streptococcus pneumoniae. After $24 \mathrm{~h}$, the cefquinome concentration dropped to less than one time the $\mathrm{MIC}_{50}$ values for most microorganisms (Table 2). The estimated $\mathrm{IC}_{50}$ against clinically important pathogens such as E. coli, S. aureus, Streptococcus agalactiae and Str. uberis was in the range from 0.001 to $4 \mu \mathrm{g} / \mathrm{mL}$ (Ehinger et al., 2006; Tenhagen et al., 2006). The concentration of cefquinome in camel calves remains above $2 \mu \mathrm{g} /$ $\mathrm{mL}$ for $12 \mathrm{~h}$ and above $1 \mu \mathrm{g} / \mathrm{mL}$ for $24 \mathrm{~h}$, which might be sufficient for treating the most sensitive microorganisms. However, microorganisms with estimated MIC values above $4 \mu \mathrm{g} / \mathrm{mL}$ require additional dose adjustments. Based on the obtained pharmacokinetic data, bacteria with MIC values of $8 \mu \mathrm{g} / \mathrm{mL}$ or higher will not be affected by the drug, as cefquinome concentrations above $4 \mu \mathrm{g} / \mathrm{mL}$ were detected only during the first $2 \mathrm{~h}$ after administration.

In conclusion, camel calves have distinct pharmacokinetic properties for cefquinome. The estimated parameters showed large deviations from the values determined in adult camels and were not in complete alignment with the values found in young animals of other species either. While the serum concentrations initially show high levels, after $24 \mathrm{~h}$ the concentration drops to about $1 \mu \mathrm{g} / \mathrm{mL}$, which might not be effective against a wide range of microorganisms. Therefore, dose adjustment taking into consideration the MIC values for the targeted microorganisms is recommended. 


\section{ACKNOWLEDGEMENT}

The authors acknowledge the Deanship of Scientific Research at King Faisal University for the financial support under Nashir Track (Grant No. 186252).

\section{REFERENCES}

Al-Taher, A. (2010): Pharmacokinetics of cefquinome in camels. J. Anim. Vet. Adv. 9, 848-852.

Amiridis, G., Fthenakis, G., Dafopoulos, J., Papanikolaou, T. and Mavrogianni, V. (2003): Use of cefquinome for prevention and treatment of bovine endometritis. J. Vet. Pharmacol. Therapeut. 26, 387-390.

Bachmann, J., Helmschrodt, C., Richter, A., Heuwieser, W. and Bertulat, S. (2018): Residue concentration of cefquinome after intramammary dry cow therapy and short dry periods. J. Dairy Sci. 101, 7540-7550.

Caprile, K. A. (1988): The cephalosporin antimicrobial agents: a comprehensive review. J. Vet. Pharmacol. Therapeut. 11, 1-32.

Champawat, M., Sankhala, L., Sharma, P. and Gaur, A. (2018): Effect of flunixin co-administration on pharmacokinetics of cefquinome following intramuscular administration in goats. Pharma. Innov. 7, 480.

Corum, O., Corum, D. D., Er, A. and Uney, K. (2019): Pharmacokinetics of cefquinome after single and repeated subcutaneous administrations in sheep. J. Vet. Pharmacol. Therapeut. 42, 647-653.

Dinakaran, V., Dumka, V. K., Ranjan, B., Balaje, R. and Sidhu, P. K. (2013): Pharmacokinetics following intravenous administration and pharmacodynamics of cefquinome in buffalo calves. Trop Anim. Health Prod. 45, 1509-1512.

Dumka, V. K., Dinakaran, V., Ranjan, B. and Rampal, S. (2013): Comparative pharmacokinetics of cefquinome following intravenous and intramuscular administration in goats. Small Rumin. Res. 113, 273-277.

Ehinger, A. M., Schmidt, H. and Kietzmann, M. (2006): Tissue distribution of cefquinome after intramammary and 'systemic' administration in the isolated perfused bovine udder. Vet. J. 172, 147-153.

El-Hewaity, M., Abd El Latif, A., Soliman, A. and Aboubakr, M. (2014): Comparative pharmacokinetics of cefquinome (Cobac$\tan 2.5 \%$ ) following repeated intramuscular administrations in sheep and goats. J. Vet. Med. 2014, 949642.

Elazab, S. T., Schrunk, D., Griffith, R. W., Ensley, S. M., Dell'Anna, G., Mullin, K., Elsayed, M. G., Amer, M. S., El-Nabtity, S. M. and Hsu, W. H. (2017): Pharmacokinetics of cefquinome in healthy and Pasteurella multocida-infected rabbits. Faseb. J. 31, lb610.

Elazab, S. T., Schrunk, D. E., Griffith, R. W., Ensley, S. M., Dell'Anna, G., Mullin, K., Elsayed, M. G., Amer, M. S., El-Nabtity, S. M. and Hsu, W. H. (2018): Pharmacokinetics of cefquinome in healthy and Pasteurella multocida-infected rabbits. J. Vet. Pharmacol. Therapeut. 41, 374-377.

Funaki, H., Uzuka, Y., Tanabe, S., Iwahara, K., Takaichi, Y., Kusano, K. -I. and Sarashina, T. (2001): Therapeutic efficacy of cefquinome against acute respiratory disease in Holstein steers. J. Jpn. Vet. Med. Assoc. 54, 451-454.

Hornish, R. E. and Katarski, S. F. (2002): Cephalosporins in veterinary medicine - ceftiofur use in food animals. Curr. Top. Med. Chem. 2, 717-731.

Hou, X. L., Wu, Y. L., Lv, Y., Xu, X. Q., Zhao, J. and Yang, T. (2013): Development and validation of an ultra-high performance liquid chromatography tandem mass spectrometry method for determination of 10 cephalosporins and desacetylcefapirin in milk. J. Chromatogr. B Analyt. Technol. Biomed. Life Sci. 931, 6-11.

Legrand, T., Vodovar, D., Tournier, N., Khoudour, N. and Hulin, A. (2016): Simultaneous determination of eight beta-lactam antibiotics, amoxicillin, cefazolin, cefepime, cefotaxime, ceftazidime, cloxacillin, oxacillin, and piperacillin, in human plasma by using ultra-high-performance liquid chromatography with ultraviolet detection. Antimicrob. Agents Chemother. 60, 4734-4742.

Limbert, M., Isert, D., Klesel, N., Markus, A., Seeger, K., Seibert, G. and Schrinner, E. (1991): Antibacterial activities in vitro and in vivo and pharmacokinetics of cefquinome (HR 111V), a new broad-spectrum cephalosporin. Antimicrob. Agents Chemother. 35, 14-19.

Murphy, S. P., Erwin, M. E. and Jones, R. N. (1994): Cefquinome (HR $111 \mathrm{~V})$ in vitro evaluation of a broad-spectrum cephalosporin indicated for infections in animals. Diagn. Microbiol. Infect. Dis. 20, 49-55.

Qu, S., Dai, C., Zhu, J., Zhao, L., Li, Y. and Hao, Z. (2018): Cefquinome-loaded microsphere formulations against Klebsiella pneumoniae infection during experimental infections. Drug Deliv. 25, 909-915.

Rana, M. P., Sadariya, K. A. and Thaker, A. M. (2015): Effect of tolfenamic acid co-administration on pharmacokinetics of cefquinome following intramuscular administration in sheep. Vet. Arh. 85, 283-292.

Shan, Q., Zhu, X., Liu, S., Bai, Y., Ma, L., Yin, Y. and Zheng, G. (2015): Pharmacokinetics of cefquinome in tilapia (Oreochromis niloticus) after a single intramuscular or intraperitoneal administration. J. Vet. Pharmacol. Therapeut. 38, 601-605.

Tenhagen, B. A., Koster, G., Wallmann, J. and Heuwieser, W. (2006): Prevalence of mastitis pathogens and their resistance against antimicrobial agents in dairy cows in Brandenburg, Germany. J. Dairy Sci. 89, 2542-2551.

Thomas, E., Roy, O., Skowronski, V., Zschiesche, E., Martin, G. and Bottner, A. (2004): Comparative field efficacy study between cefquinome and gentamicin in neonatal calves with clinical signs of septicaemia. Rev. Med. Vet. 155, 489-493.

UCT Application Note 5109-02-01 (2014): A determination of veterinary drug residues in milk using polymeric SPE and UHPLC-MS/MS analysis.

Uney, K., Altan, F., Altan, S., Erol, H., Arican, M. and Elmas, M. (2017): Plasma and synovial fluid pharmacokinetics of cefquinome following the administration of multiple doses in horses. J. Vet. Pharmacol. Therapeut. 40, 239-247.

Uney, K., Altan, F., Cetin, G., Aboubakr, M., Dik, B., Sayin, Z., Er, A. and Elmas, M. (2018): Pharmacokinetics of cefquinome in red-eared slider turtles (Trachemys scripta elegans) after single intravenous and intramuscular injections. J. Vet. Pharmacol. Therapeut. 41, e40-e44. 
Uney, K., Altan, F. and Elmas, M. (2011): Development and validation of a high-performance liquid chromatography method for determination of cefquinome concentrations in sheep plasma and its application to pharmacokinetic studies. Antimicrob. Agents Chemother. 55, 854-859.

Venkatachalam, D., Dumka, V. and Ranjan, B. (2018a): Pharmacokinetics of a single intramuscular injection of cefquinome in buffalo calves. J. Vet. Pharmacol. Therapeut. 41, 155-158.

Venkatachalam, D., Dumka, V. K. and Ranjan, B. (2018b): Pharmacokinetics of a single intramuscular injection of cefquinome in buffalo calves. J. Vet. Pharmacol. Therapeut. 41, 155-158.

Wang, J., Fan, X., Liu, Y., Du, Z., Feng, Y., Jia, L. and Zhang, J. (2017): Extraction optimization of sixteen cephalosporins in milk by filtered solid phase extraction and ultra-high pressure liquid chromatography coupled to tandem mass spectrometry. Anal. Methods 9, 1282-1289.

Yu, Y., Zhou, Y. F., Chen, M. R., Li, X., Qiao, G. L., Sun, J., Liao, X. P. and Liu, Y. H. (2016): In vivo pharmacokinetics/pharmacodynamics of cefquinome in an experimental mouse model of Staphylococcus aureus mastitis following intramammary infusion. PLoS One 11, e0156273.
Zhang, L., Wu, X., Huang, Z., Kang, Z., Chen, Y., Shen, X., Cai, Q. and Ding, H. (2019): Pharmacokinetic/pharmacodynamic integration of cefquinome against Pasteurella multocida in a piglet tissue cage model. J. Vet. Pharmacol. Therapeut. 42, 60-66.

Zhang, L., Wu, X., Huang, Z., Zhang, N., Wu, Y., Cai, Q., Shen, X. and Ding, H. (2018): Pharmacokinetic/pharmacodynamic assessment of cefquinome against Actinobacillus pleuropneumoniae in a piglet tissue cage infection model. Vet. Microbiol. 219, 100-106.

Zhang, Y., Huo, M., Zhou, J. and Xie, S. (2010): PKSolver: an addin program for pharmacokinetic and pharmacodynamic data analysis in Microsoft Excel. Comput. Methods Progr. Biomed. 99, 306-314.

Zhao, D. H., Wang, X. F., Wang, Q. and Li, L. D. (2017): Pharmacokinetics, bioavailability and dose assessment of cefquinome against Escherichia coli in black swans (Cygnus atratus). BMC Vet. Res. 13, 226.

Zhou, Y. F., Zhao, D. H., Yu, Y., Yang, X., Shi, W., Peng, Y. B. and Liu, Y. H. (2015): Pharmacokinetics, bioavailability and PK/PD relationship of cefquinome for Escherichia coli in Beagle dogs. J. Vet. Pharmacol. Therapeut. 38, 543-548. 